\title{
Images of the month 1: Cough before the storm: A case of pulmonary alveolar microlithiasis
}

\author{
Authors: Momina F Yazdani, ${ }^{\mathrm{A}}$ Deborah Morris-Rosendahl, ${ }^{\mathrm{B}}$ Lucia Chen, ${ }^{\mathrm{C}}$ Anand Devaraj ${ }^{\mathrm{D}}$ and Felix Chua $^{\mathrm{E}}$
}

KEYWORDS: Pulmonary alveolar microlithiasis, SLC34A2 gene

\section{Case presentation}

A 45-year-old woman, referred for assessment of a chronic dry cough, underwent plain chest X-ray which demonstrated innumerable small pulmonary nodules producing a 'sandstorm' appearance (Fig 1). She was constitutionally well and not dyspnoeic. The cough had subsided considerably by the time of her review. Tests for active tuberculosis, autoimmune disorders and thyroid disease were negative. High-resolution computed tomography showed widespread interlobular septal thickening and a dense micronodular pattern consistent with pulmonary alveolar microlithiasis (PAM; Fig 2).

Genetic testing confirmed that the patient was homozygous for a pathogenic frameshift variant in the SLC34A2 gene: c.1653_1660del, which results in a termination codon, p.(Trp552AlafsTer109). The protein encoded by this gene is involved in phosphate homeostasis and alveolar surfactant metabolism. Although a family history of lung disease was not obtained, she reported parental consanguinity.

Pulmonary function testing demonstrated a mild restrictive ventilatory defect with slight gas transfer impairment. Regular monitoring was instituted and a referral made to the regional thoracic transplant assessment service.

\section{Discussion}

PAM is a rare autosomal recessive disease characterised by the accumulation of innumerable calcium phosphate deposits known as microliths in the lungs. It is caused by pathogenic variants in the SLC34A2 gene which is located on chromosome 4 p15 and encodes a 690-amino acid type IIb sodium phosphate cotransporter. This protein is expressed in alveolar type II cells and its mutation results

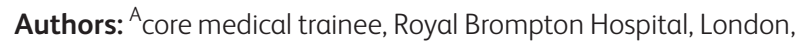
UK; ${ }^{B}$ consultant clinical geneticist, Royal Brompton Hospital, London, UK; ' ${ }^{C}$ core medical trainee, Royal Brompton Hospital, London, UK;

${ }^{D}$ consultant radiologist, Royal Brompton Hospital, London, UK; ${ }^{E}$ consultant respiratory physician, Royal Brompton Hospital, London UK

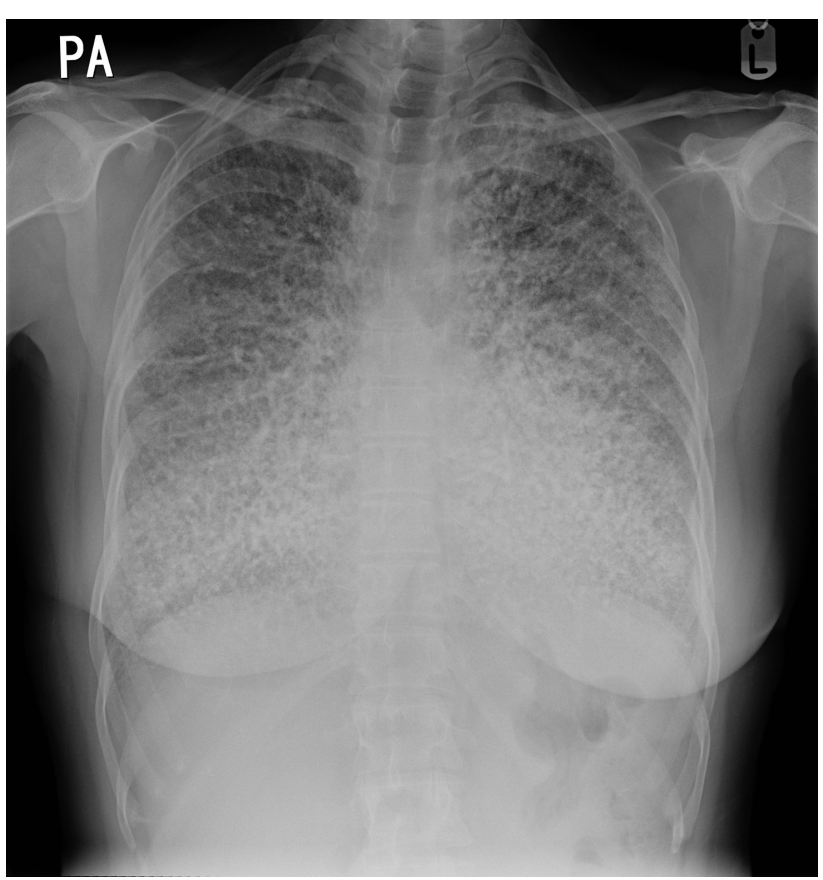

Fig 1. Chest X-ray showing widespread pulmonary calcification suggesting a 'sandstorm' appearance.

in disordered phosphate homeostasis and clearance from the alveoli.

The extensive 'sandstorm' calcification in PAM is typically an incidental X-ray finding which precedes the onset of significant symptoms. ${ }^{2}$ Genetic testing confirms PAM, a diagnosis usually made in childhood to the 4 th decade of life. Familial occurrence is seen in a third of cases and there is also a strong association within consanguineous families. ${ }^{1}$

While patients are often minimally affected at the time of diagnosis, regular surveillance is important as progression of the condition can result in ventilatory failure secondary to extensive microlithiasis, pulmonary fibrosis, pneumothorax or cor pulmonale. ${ }^{2}$ To date, lung transplant remains the only effective therapeutic option. 
Fig 2. Axial lung window high-resolution computed tomography. a) Axial lung window setting demonstrating extensive interlobular septal thickening and $a$ micronodular pattern. b) Axial wide-window setting reveals extensive high-density calcified spherules consistent with pulmonary alveolar microlithiasis.
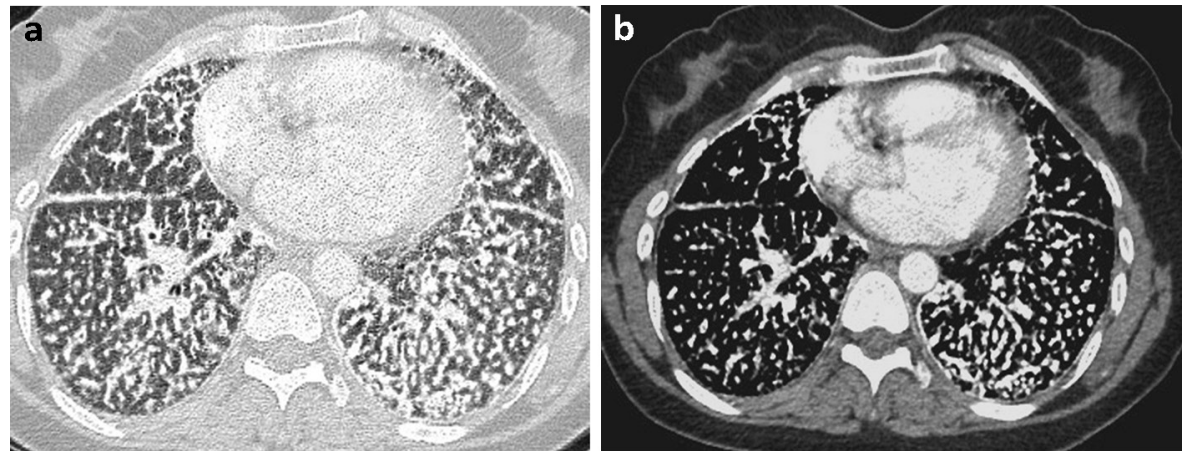

\section{References}

1 Castellana G, Castellana G, Gentile M, Castellana R, Resta O. Pulmonary alveolar microlithiasis: review of the 1022 cases reported worldwide. Eur Respir Rev 2015;24:607-20.

2 Saito A, McCormack FX. Pulmonary alveolar microlithiasis. Clin Chest Med 2016;37:441-8.

Address for correspondence: Dr Felix Chua, Respiratory Department, Fulham Wing, Royal Brompton Hospital, Dovehouse St, Chelsea, London SW3 6JY, UK. Email: f.chua@rbht.nhs.uk

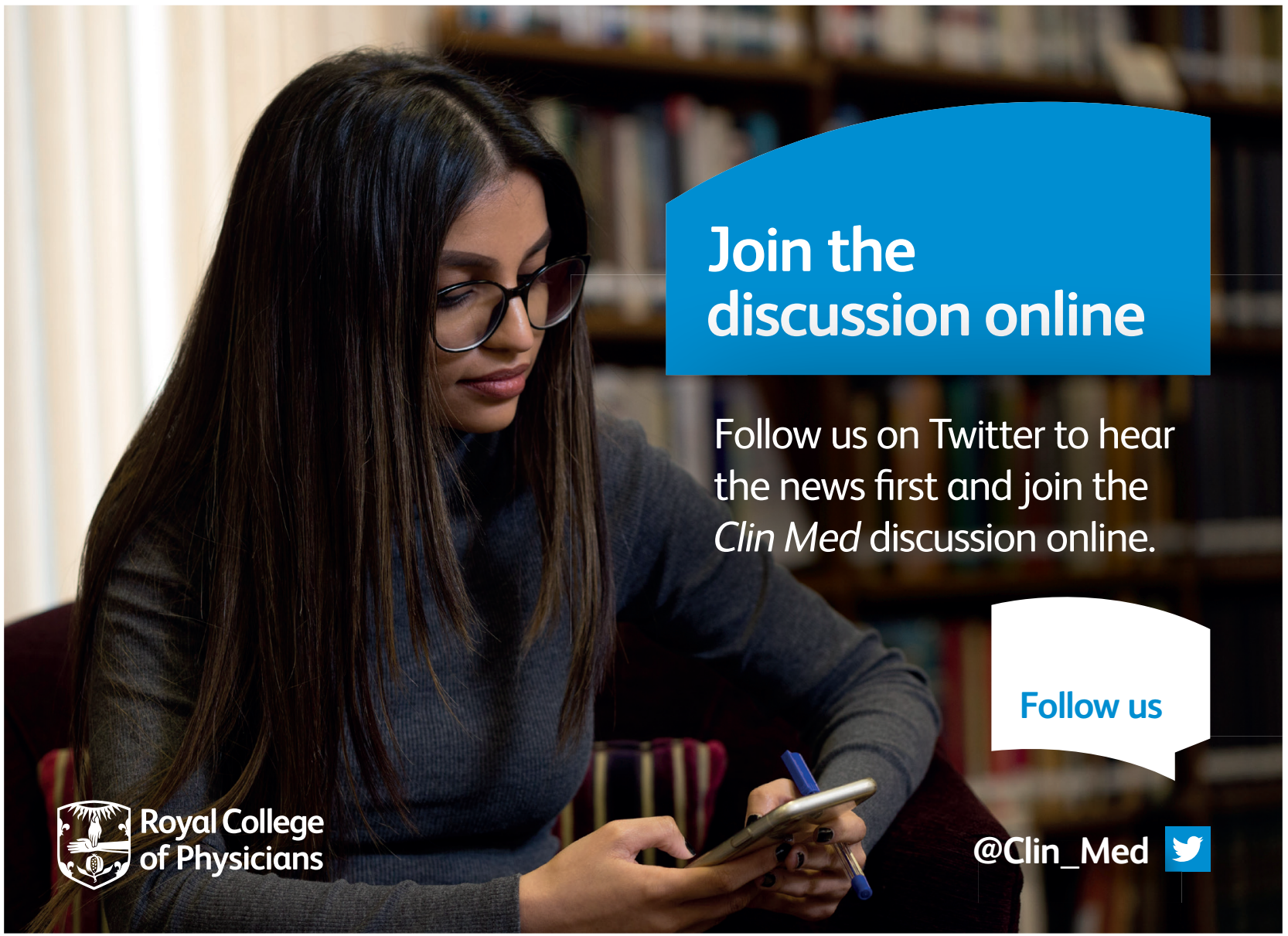

